\title{
A new global malaria eradication strategy
}

Richard Feachem, Oliver Sabot

On Oct 17, 2007, Bill and Melinda Gates called for complete eradication to be adopted as the new goal for the age-old fight against malaria, with the Director General of WHO, Margaret Chan, promptly echoing their conviction. Although debate over the wisdom of this target will continue, growing impatience with the low ambitions of current efforts, fuelled by reductions in morbidity and mortality in some countries ${ }^{1}$ and progress in the development of new drugs and the first-ever vaccine, will lead many decision makers to adopt eradication of malaria as the primary aim for their organisations.

Two crucial questions stand out for those organisations that will now begin striving towards malaria eradication. When and how can it be achieved? Barring a magic bullet, which the most promising vaccine candidates are not, ${ }^{2}$ even the most optimistic malaria experts agree that eradication is decades away. The latter question, however, requires prompt attention.

If a goal as ambitious as eradication is to be achieved, key groups-including donors, technical agencies, the scientific community, and countries where the disease is endemic-must align their energies and resources behind a common approach. That strategy should not be so prescriptive as to prevent appropriate variation among countries and donors, but should provide strong direction to ensure that global investment is well targeted and coordinated. Alternatively, if each donor and agency pursues their own agenda, the momentum gathered over the past 5 years will be lost and the fatalism and inaction that characterised the last decades of the 20th century will return. ${ }^{3}$

The malaria community should therefore take immediate steps, including broad debate and detailed technical consultation, to develop a common approach. In November, 2007, the Roll Back Malaria Partnership endorsed the creation of a global malaria business plan to guide collective eradication efforts. With this Viewpoint, we hope to begin a debate on the strategy that can provide the best overarching framework for that plan and often collective malaria efforts.

The first global strategy for the fight against malaria was adopted in 1955 at the start of the now notorious Global Malaria Eradication Program. This strategy called for massive and rapid application of dichlorodiphenyltrichloroethane (DDT) to interrupt transmission of the disease in countries around the world, regardless of geography and epidemiology (the notable exception was the exclusion of sub-Saharan Africa from this, so called, global strategy). ${ }^{4}$ This approach failed to interrupt transmission completely in many countries and malaria resurged to previous or even higher levels as eradication programmes crumbled and the strategy was abandoned. ${ }^{5}$
Although no-one will argue for the resurrection of this strategy in full, current impatience has revived interest in rapid indiscriminate attacks to eradicate malaria.

Countries such as Kenya, Rwanda, and Zambia, which generated enthusiasm for an all-out attack with success in reducing malaria mortality by as much as $60 \%$, also show why this strategy would not be advisable. Even if one of these countries did successfully eliminate transmission of malaria, they would find it nearly impossible to sustain, because the parasite would inevitably be reintroduced by migrants and travellers from neighbouring countries with high transmission. Both experience and modelling show us that even a few infections can quickly lead to an epidemic in areas with efficient vectors and limited protective measures. ${ }^{5}$ One of the principal lessons of the first global eradication campaign was that the intensive effort needed to prevent resurgence in areas with continued exposure to parasite reservoirs cannot be maintained over time, because field workers tire and lose precision and donors and governments shift resources to seemingly more urgent problems. ${ }^{6}$ Thus, even with the large arsenal and war chest available today, an indiscriminate push to eliminate malaria could lead to epidemics and erosion of years of work and investment.

Today's efforts against malaria target a reduction in malaria mortality through progressive scaling up of a package of interventions. Progress in implementation was at first poor. In the past 3 years, however, many countries have reported more promising results, with steep reductions in morbidity through increased use of insecticide-treated bed nets and other interventions. ${ }^{8,9}$ Seeking to expand this success, a new report launched at the 2008 World Economic Forum in Davos compellingly makes the case for a concerted short-term push to increase coverage of key interventions above $80 \%$ and slash morbidity and mortality in malaria-endemic countries. ${ }^{10}$

Aggressive scaling up should be the central component of any new strategy, with a dedicated group of well managed professionals helping countries to overcome bottlenecks and to achieve their goal. But the Davos report and other descriptions of this approach leave an essential question unanswered: what happens after aggressive short-term targets are achieved? Maintainence of intensive interventions will be difficult once malaria is no longer a major public-health threat and donors and populations lose interest. A breakthrough intervention, such as a highly protective and long-lasting vaccine, will not be available for at least 20 years, while widespread changes in living standards of the sort that contributed to elimination in Europe and the USA will undoubtedly take much longer. ${ }^{11}$ Hence, a short-term push must be complemented by a long-term strategy.
Lancet 2008; 371: 1633-35

Published Online March 27, 2008 DOI:10.1016/S0140 6736(08)60424-9

See Lancet Infectious Diseases DOI:10.1016/S14733099(08)70045-8

Clinton Foundation HIV/AIDS Initiative, New York, NY, USA (O Sabot BA); and Global Health Group, University of California, San Francisco, CA, USA (Sir R Feachem DSc[Med]) Correspondence to: Mr Oliver Sabot, Clinton Foundation HIV/AID Initiative, Quincy, MA 02169, USA osabot@clintonfoundation. org 
In this Viewpoint, we outline a new global malaria strategy with two main components. First, control efforts would be intensified in the heartland of the malaria endemic - the holoendemic and hyperendemic regions of tropical Africa, Asia, and Latin America. Second, and currently, countries at the margins of the endemic zone would attempt to completely interrupt transmission of the disease at the same time. Over time, as each country eliminates transmission, one or more of their neighbours would begin to pursue elimination with the newly malaria-free area as a foundation, benefiting from the reductions in transmission achieved by the intensified control effort. This process would continue until malaria was eradicated or new technology enabled a shift in strategy.

Malaria has natural margins determined largely by latitude, altitude, climate, and, in some cases, successful human intervention. As latitude and altitude increase, transmission becomes less efficient and malaria less prevalent. ${ }^{12}$ This strategy targets elimination at the current limits of malaria where interruption of transmission is most feasible. The initial target areas, or fronts, could include the southernmost margins of endemic malaria in Africa (Botswana, Namibia, South Africa, and Swaziland), the natural limits of Anopheles mosquitoes in the Pacific islands (the Solomon Islands and Vanuatu), and the northernmost malaria margins in Asia (China) and the Americas (southern Mexico). In addition to their epidemiological advantages, the non-Melanesian countries listed have greater wealth and stability and stronger health systems than most malaria-endemic nations. ${ }^{13}$

In addition to its technical feasibility, the greatest advantage of pursuing elimination from the margins is the reduced risk of resurgence. Prevention of reintroduction is always challenging, but it is near impossible for a country that has porous borders with several countries with endemic malaria. The countries that would initially target elimination have one or two borders with other malaria endemic areas and commonly have large sections of their territory that are already free of the disease. However, even one border with a malaria-endemic area can undermine elimination, and many of the countries that might be targeted border low-income areas with high malaria transmission. For example, Namibia would have to prevent reintroduction from southern Angola. Effectively rising to this challenge will require robust cross-border collaboration. Fortunately, there is a strong incentive for such collaboration: joint investment in malaria control in southern Angola will yield significant returns for both nations. The successful Lubombo Spatial Development Initiative between Mozambique, South Africa, and Swaziland has proven the potency of such collaborations and spurred similar approaches in the region, such as the nascent Trans-Zambezi Initiative. $^{14,15}$
Systematically eliminating malaria from its current margins will gradually expand areas without malaria transmission, freeing millions of people from the threat of infection and death and stimulating investment and tourism. This approach not only makes elimination more feasible to achieve and sustain but also is economically attractive. Large initial investments in elimination would be offset by lower ongoing costs: preventing reintroduction across just one border will cost less than indefinitely maintaining control interventions at high levels. However, relative cost-effectiveness of control and elimination programmes is poorly understood and more robust analysis, taking into account factors such as effect on tourism and investment, should be a research priority.

The strategy of gradually eliminating malaria has begun to emerge organically without any signal or recognition from the global community. The Solomon Islands and Vanuatu, with the support from the Australian Government, have recently launched a stepwise approach to interrupt malaria transmission..$^{16}$ In March 2007, Ministers of Health from the Southern Africa Development Community approved a new regional malaria strategy that includes a target of eliminating malaria from six member nations by $2015 .{ }^{17}$ Interest in and political support for malaria elimination has independently emerged in other countries with favourable conditions, including the Comoros and Madagascar. Irrespective of whether the global community codifies their actions in an official strategy, these countries should be lauded for their ambition and provided with appropriate support.

The greatest potential shortcoming of this strategy is that donors, attracted by their novelty and ambitious objectives, might invest in elimination programmes at the expense of under-resourced control efforts. Elimination will always cost more per case than control in an endemic area and will require a portion of the finite pool of international support. However, international funding for malaria will probably continue to grow, making potential trade-offs between control and elimination less severe. Furthermore, returns on investment in control will be much lower in the long run in the absence of elimination or another means to consolidate the gains made. As such, the malaria community should focus on the appropriate distribution of resources between both control and elimination rather than either alone, with detailed guidance for donors and countries alike.

This strategy and malaria elimination in resource-poor areas needs more thought and discussion. What interventions should be used in areas with efficient vectors and weak health systems? How will Plasmodium vivax be eliminated after $P$ falciparum? How can malaria resurgence be effectively prevented in sub-Saharan Africa, and how much will this cost? These and many other questions should be answered, drawing 
on the lessons learned from the first eradication campaign and other experiences. The Malaria Elimination Group, a gathering of international malaria experts convened by the Global Health Group of the University of California, San Francisco, had its first meeting in Santa Cruz, CA, USA, on March 23-26, to explore these questions and to develop a prospectus for malaria elimination. But although important, this process is not sufficient. An effort as large and complex as malaria elimination requires input from many researchers and practitionersinput that is largely absent today. 40 years ago, WHO had 500 staff focusing on malaria elimination ${ }^{5}$ - today there is only a handful around the world. This gap must be closed and more resources devoted to research and implementation support for elimination efforts to be successful.

This approach is only one possible path to malaria eradication. We hope that this Viewpoint will inspire debate on the most appropriate strategy to achieve eradication and that others will rapidly come forward with new proposals and evidence. We have left the topic of research largely untouched because it is essential to any strategy that might be adopted. To eradicate malaria, we will need new drugs, insecticides, and other interventions as well as increased understanding of the parasites and vectors. Any final strategy should recognise this crucial role for improved research and provide a framework for how that should be done and financed.

Although a robust strategy is important, the success of malaria eradication will ultimately be determined by financial and political commitment, sound management, and, the work of tens of thousands of individuals around the world. We must, therefore, quickly decide on a new strategy and shift the full attention and resources of the global community to the aggressive implementation of programmes in endemic areas. If we do so with sufficient courage, conviction, and speed, we may be able to soon resume rolling back the burden of malaria across the world.
Conflict of interest statement

We declare that we have no conflict of interest.

References

1 Fegan GW, Noor AM, Akhwale WS, Cousens S. Effect of expanded insecticide-treated bed net coverage on child survival in rural Kenya: a longitudinal study. Lancet 2007; 370: 1035-39.

2 Aponte J, Aide P, Renom M, Mandomando I. Safety of the RTS,S/AS02D candidate malaria vaccine in infants living in a highly endemic area of Mozambique: a double blind randomized controlled phase I/IIb trial. Lancet 2007; 370: 1543-51.

3 Feachem RGA, Sabot OJ. Global malaria control in the 21st century: A historic but fleeting opportunity. JAMA 2007; 297: 2281-84.

4 World Health Organization. Ninth world health assembly. Int Organ 1956; 10: 642-44.

5 Harrison G. Mosquitoes, malaria, and man: a history of the hostilities since 1880. New York: EP Dutton, 1978.

6 Choudhury DS. Malaria in India: past, present and future. Indian J Pediatr 1985; 52: 243-48.

7 Feachem RGA, Daniels D, Dunlop D, Medlin C. Final report of the external evaluation of Roll Back Malaria. London: Malaria Consortium, 2002.

8 Nyarango PM, Gebremeskel T, Mebrahtu G, Mufunda J. A steep decline of malaria morbidity and mortality trends in Eritrea between 2000 and 2004: the effect of combination of control methods. Malar J 2006; 5: 33.

9 UNICEF, Roll Back Malaria Partnership. Malaria and children: progress in intervention coverage. New York: UNICEF, 2007.

10 Malaria No More. We can't afford to wait: the business case for the rapid scale-up of malaria control in Africa. New York: Malaria No More and McKinsey \& Company, 2008.

11 Humphreys M. Malaria: poverty, race, and public health in the United States. Baltimore: Johns Hopkins University Press, 2001.

12 Snow RW, Guerra CA, Noor AM, Myint HY. The global distribution of clinical episodes of Plasmodium falciparum malaria. Nature 2005; 434: 214-17.

13 World Health Organization. Malaria elimination: a field manual for low and moderate endemic countries. Geneva: World Health Organization, 2007.

14 Sharp B, Kleinschmidt I, Streat E, Maharaj R. Seven years of regional malaria control collaboration-Mozambique, South Africa, Swaziland. Am J Trop Med Hyg 2007; 76: 42-47.

15 AllAfrica.com. Namibia: cross-border initiative launched against malaria. November 22, 2006. Washington, DC. http://allafrica.com/ stories/200611200207.html.

16 Pacific Malaria Initiative. Action plans for the Solomon Islands and Vanuatu: unpublished papers presented to the 2nd meeting of the Malaria Reference Group. Port Vila, Vanuatu, December 5, 2007.

17 Southern Africa Development Community. SADC malaria strategic plan 2007-2015. Gaborone, Botswana, 2007. 